\title{
Análisis del perfil de flexibilidad en jóvenes taekwondistas
}

\author{
Antonio CEJUDO PALOMO*1 (D), Blanca SAN CIRILO SORIANO², Francisco Javier ROBLES \\ PALAZÓN $^{1}$, Mํㅡㄹ del Pilar SAIZ DE BARANDA ${ }^{1}$ \\ ${ }^{1}$ Facultad de Ciencias del Deporte. Universidad de Murcia (España) \\ 2 Universidad Miguel Hernández de Elche (España)
}

I Congreso Nacional de Entrenadores de Judo, Murcia (España), 13-15 de julio, 2018

\begin{abstract}
Resumen
El objetivo principal del presente estudio fue determinar el perfil de flexibilidad de la extremidad inferior en atletas jóvenes de taewwondo, así como identificar los deportistas con cortedad muscular. Para ello, un total de 17 taekwondistas jóvenes participaron en el presente estudio; siete deportistas pertenecían a la selección española y diez a la selección Murciana. El rango de movimiento de los principales movimientos de la extremidad inferior fue valorado mediante el protocolo "ROM SPORT" y se realizó un análisis descriptivo de cada una de las variables cuantitativas. Se aplicó la prueba t-test de Student o Wilcoxon dependiendo de la normalidad de los datos. Los valores de ROM de cada movimiento se clasificaron como "normal" o "cortedad" de acuerdo con valores de referencia previamente justificados.

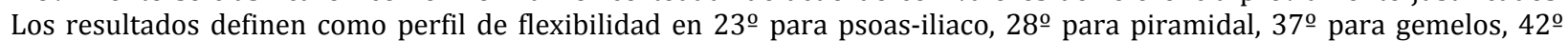

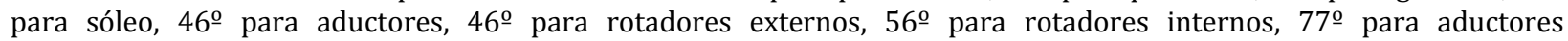
monoarticulares, $80^{\circ}$ para isquiosurales, $140^{\circ}$ para cuádriceps y $145^{\circ}$ para glúteo mayor. La medición individual del ROM identificó cortedad muscular en 11 deportistas en piramidal (64,7\%), 13 deportistas en sóleo (76,4\%), 8 deportistas en rotadores externos (47\%), 14 deportistas en aductores monoarticulares (82,3\%), 13 deportistas en isquiosural (76,4\%), 4 deportistas en cuádriceps $(23,5 \%)$ y 12 en glúteo mayor $(70,5 \%)$. Este perfil de flexibilidad puede ayudar a los profesionales del deporte a marcar objetivos cuantificables en el entrenamiento de la flexibilidad en este deporte. La inclusión de estiramientos, o el aumento de la dosis deben de aplicarse sobre las cortedades identificadas en piramidal, sóleo, rotadores externos de cadera, aductores monoarticulares, isquiosural, cuádriceps y glúteo mayor.
\end{abstract}

Palabras clave: Deportes de combate; taekwondo; rango de movimiento; cortedad; lesión.

\begin{abstract}
The main aim of this study was to determine the flexibility profile of the lower extremity in young taekwondo athletes, as well as identifying athletes with muscle shortness. For this purpose, a total of 17 young taekwondo athletes were assessed; seven from the Spanish team and ten from the Murcia team. The range of motion of the main movements of the lower extremity was evaluated by the "ROM SPORT" protocol and a descriptive analysis of each of the quantitative variables was performed. A Student $t$-test or arWilcoxon test was applied depending on the normality distribution of the data. The ROM values of each movement were classified as "normal" or "shortness" according to previously determined reference values. The results define the following flexibility profile: $23^{\circ}$ for iliopsoas, $28^{\circ}$ for piriformis, 37으 for gastrocnemius, $42^{\circ}$ for soleus, $46^{\circ}$ for adductors, $46^{\circ}$ for external rotators, $56^{\circ}$ for internal rotators, $77^{\circ}$ for monoarticular adductors, $80^{\circ}$ for hamstrings, $140^{\circ}$ for quadriceps and 145을 for gluteus maximus. Individual evaluations identified muscle shortness in pyramidal (11 athletes, 64.7\%), soleus (13 athletes, 76.4\%), external rotators (8 athletes, $47 \%$ ), monoarticular adductors (14 athletes, 82.3\%), hamstrings (13 athletes, $76.4 \%$ ), quadriceps ( 4 athletes, $23.5 \%$ ) and gluteus maximus (12 athletes, 70.5\%). This flexibility profile can help sports professionals to set quantifiable goals for the training of flexibility in this sport. The inclusion of stretching exercises or the increase of their doses should be applied for the improvement of the identified shortness in piriformis, soleus, external hip rotators, monoarticular, hamstring, quadriceps and gluteus maximus adductors.
\end{abstract}

Analysis of the flexibility profile in young taekwondo athletes

Keywords: Combat sports; taekwondo; range of movement; tightness; injury.

\section{Introducción}

El taekwondo actualmente es un deporte de combate olímpico con cerca de 70 millones de practicantes en 208 países (World Taekwondo Federation - WTF, 2017). Los deportistas de taekwondo precisan un bajo porcentaje de grasa corporal, niveles moderados a altos de consumo

*Email: acpcejudo@gmail.com 
máximo de oxígeno, alta potencia anaeróbica, moderada fuerza explosiva y alta velocidad de reacción y velocidad gestual en las extremidades inferiores, indispensable para un óptimo rendimiento físico-técnico en situaciones de defensa y contraataques (Bridge et al., 2014; Cardozo et al., 2017). La naturaleza dinámica de las acciones técnicas y tácticas del taekwondo exigen una gran flexibilidad de las extremidades inferiores (Wasik, 2009; Pion, Fransen, Lenoir, \& Segers, 2014). Sin embargo, se han encontrado escasos trabajos que analicen el perfil de flexibilidad de los practicantes federados de este deporte. Se requiere una investigación más extensa en las características físicas y fisiológicas de los atletas de taekwondo para determinar el rendimiento óptimo con menor predisposición a la lesión deportiva. El objetivo principal del presente estudio fue determinar el perfil de flexibilidad de la extremidad inferior en atletas jóvenes de taekwondo, así como identificar los atletas con cortedad muscular.

\section{Método}

\subsection{Muestra}

Un total de 17 taekwondistas jóvenes participaron en el presente estudio; siete deportistas $(18,1 \pm 3,2$ años; $66,6 \pm 10,2 \mathrm{~kg} ; 178 \pm 6,8 \mathrm{~cm})$ pertenecían a la selección española y diez deportistas a

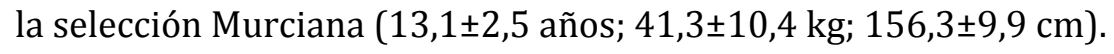

\subsection{Procedimiento}

Se valoraron los siguientes rangos de movimiento en la extremidad dominante y no dominante, siguiendo la metodología que describen Cejudo, Sainz de Baranda, Ayala y Santonja (2015): extensión de cadera (EC) para el psoas iliaco, aducción de cadera con rodilla flexionada (ADC) para los músculos abductores (piramidal, glúteo medio y glúteo menor), dorsi-flexión del tobillo con rodilla extendida (DFT_RE) para el gemelo, dorsi-flexión del tobillo con rodilla flexionada (DFT_RF) para el sóleo, abducción de cadera con rodilla extendida (ABC) para los aductores (pectíneo, aductor corto, aductor mediano o largo, aductor mayor y recto interno), rotación interna de cadera (RIC) para los músculos rotadores externos, rotación externa de cadera (REC) para los músculos rotadores internos, abducción de cadera con la rodilla flexionada (ABC_CF) para los abductores monoarticulares (pectíneo, aductor corto, aductor mediano y aductor mayor), flexión de cadera con rodilla extendida (FC_RE) para los isquiosurales, flexión de rodilla (FR) para el cuádriceps y flexión de cadera con rodilla flexionada (FC) para el glúteo mayor. Los resultados de esta medición definen el perfil de flexibilidad de la extremidad inferior, que forman parte de la versión completa del protocolo ROM-SPORT (Cejudo, 2015).

Después del calentamiento, y en orden aleatorio, se llevó a cabo el protocolo de valoración ROM SPORT. De cada medida se realizaron dos valoraciones máximas y la media de ambas se utilizó en el análisis estadístico. Los valores de ROM se clasificaron como "normal versus cortedad", de acuerdo a los valores de referencia propuestos para considerar que un deportista es más propenso

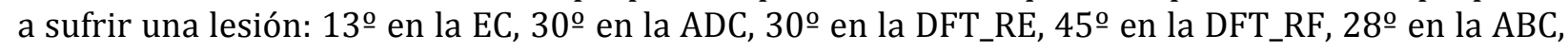
$45^{\circ}$ en la RIC, $50^{\circ}$ en la REC, $80^{\circ}$ en la $\mathrm{ABC} \_\mathrm{CF}, 88,1^{\circ}$ en la FC_RE, $132^{\circ}$ en la FR y $135^{\circ}$ en la FC (tomado de Cejudo et al., 2017). Por otro lado, los puntos de corte que se utilizaron para la asimetría fueron 6을 para los ROM pequeños (EC, ADC, DFT_RE, DFT_RF, ABC, RE y RIC) y $10^{\circ}$ para los ROM más grandes (RT, ABC_CF, FC_RE, FR y FC) (Ellenbecker et al, 2007; López-Valenciano et al., en prensa).

\subsection{Análisis estadístico}

Se realizó un análisis descriptivo de cada una de las variables cuantitativas, que incluía la media y su correspondiente desviación típica, para las medidas ROM de cadera, rodilla y tobillo. Para examinar la existencia de asimetría entre los valores de los lados dominante y no dominante, se utilizó la prueba t-test de Student (si la distribución de los datos fue normal) y la prueba de Wilcoxon (si la distribución de los datos no fue normal). Además, se calculó el tamaño del efecto de Cohen de todos los resultados, y la magnitud del efecto era interpretado de acuerdo con los 
criterios de Hopkins, Marshall, Batterham y Hanin (2009), en el cual un tamaño de efecto menos de 0,2 , de 0,2 a 0,59 , de 0,6 a 1,19 , de 1,20 a 2,00, de 2,00 a 3,99 y superior a 4,00 era considerado como trivial, pequeño, moderado, grande, muy grande y extremadamente grande, respectivamente. Los autores decidieron arbitrariamente "moderado" como el nivel mínimo de efecto relevante con aplicación práctica en los resultados. Una prueba $U$ de Mann-Whitney (datos no paramétricos) o $t$ test para muestras independiente (datos paramétricos) fue utilizada para determinar la existencia de diferencias entre los valores medios de los grupos normal y cortedad en cada uno de los movimientos valorados. El tamaño del efecto de cada variable se analizó utilizando el estadístico $r$ de Pearson entre los grupos (Cortedad vs normal) (0,0-0,39 = efecto bajo, 0,4-0,69 efecto medio y 0,7-1 efecto alto) (Pallant, 2007).

\section{Resultados}

Los resultados definen como perfil de flexibilidad en $23^{\circ}$ para psoas-iliaco, $28^{\circ}$ para piramidal, $37^{\circ}$ para gemelos, $42^{\circ}$ para sóleo, $46^{\circ}$ para aductores, $46^{\circ}$ para rotadores externos, $56^{\circ}$ para rotadores internos, $77^{\circ}$ para aductores monoarticulares, $80^{\circ}$ para isquiosurales, $140^{\circ}$ para cuádriceps y 145으 para glúteo mayor. La medición individual del ROM identificó cortedad muscular a 11 deportistas en piramidal (64,7\%), 13 deportistas en sóleo (76,4\%), 8 deportistas en rotadores externos (47\%), 14 deportistas en aductores monoarticulares, 13 deportistas en isquiosural (76,4\%), 4 deportistas en cuádriceps $(23,5 \%)$ y 12 en glúteo mayor $(70,5 \%)$.

\section{Discusión}

El taekwondo es un deporte dinámico en la que los gestos técnicos requieren un gran ROM, especialmente en las extremidades inferiores (Wasik, 2009). A pesar de la importancia de esta cualidad física en el rendimiento físico-técnico de este deporte, son escasos los estudios que han valorado la flexibilidad en taekwondistas. Además, los autores de estos trabajos han utilizado especialmente la prueba de valoración lineal (centímetros) "test distancia de dedos planta" para medir la extensibilidad isquiosural. Los valores encontrados en este test para taekwondistas internacionales sénior oscilaron entre 36-36.9 cm para los hombres y 35.2-56.6 cm para las mujeres (Campos et al., 2012; Heller et al., 1998; Markovic, Misigoj-Durakovic y Trninic, 2005; Rivera, Rivera-Brown y Frontera, 1998). Estos valores no deben ser comparados con el test angular FC_RE del presente estudio, aunque ambos tests miden la extensibilidad de la misma musculatura. La influencia de las medidas antropométricas de los taekwondistas, la ejecución bilateral del tests y el significativo grado de retroversión pélvica resulta en una mayor extensibilidad isquiosural, que afecta a la validez interna de la medida.

\section{Conclusiones}

Este perfil de flexibilidad puede ayudar a los profesionales del deporte a marcar objetivos cuantificables en el entrenamiento de la flexibilidad en este deporte. La inclusión de estiramientos, o el aumento de la dosis deben de aplicarse sobre las cortedades identificadas en piramidal, sóleo, rotadores externos de cadera, aductores monoarticulares, isquiosural, cuádriceps y glúteo mayor.

\section{Referencias}

Bridge, C.A., Ferreira da Silva Santos, J., Chaabène, H., Pieter, W., \& Franchini, E. (2014). Physical and physiological profiles of taekwondo athletes. Sports Medicine, 44(6), 713-33. doi: 10.1007/s40279-014-0159-9

Campos, F.A., Bertuzzi, R., Dourado, A.C., Santos, V.G.F., \& Franchini, E. (2012). Energy demands in taekwondo athletes during combat simulation. European Journal of Applied Physiology, 112(4), 1221-1228. doi: 10.1007/s00421-011-2071-4 
Cardozo, L. A., Vera-Rivera, D. A., Conde-Cabezas, O. A. y Yánez, C. A. (2017). Aspectos fisiológicos de deportistas elite de taekwondo: Una revisión narrativa. Revista Española de Educación Física y Deportes, (418), 35-46.

Cejudo, A. (2015). Deporte y flexibilidad: Rendimiento deportivo sin riesgo de lesión (Tesis Doctoral). Universidad de Murcia, San Javier, España.

Cejudo, A., Sainz de Baranda, P., Ayala, F. \& Santonja, F. (2015). Test-retest reliability of seven common clinical tests for assessing lower extremity muscle flexibility in futsal and handball players. Physical Therapy in Sport, 16(2), 107-113. doi: 10.1016/i.ptsp.2014.05.004

Cejudo, A., Sainz de Baranda, P., Ayala, F. \& Santonja, F. (2017). Clasificación de los valores de rango de movimiento de la extremidad inferior en jugadores de fútbol sala. SPORT TK, 6(1), 41-50.

Ellenbecker, T.S., Ellenbecker, G.A., Roetert, E.P., Silva, R.T., Keuter, G. \& Sperling, F. (2007). Descriptive profile of hip rotation range of motion in elite tennis players and professional baseball pitchers. The American Journal of Sports Medicine, 35(8), 1371-1376.

Heller, J., Peric, T., Dlouha, R., Kohlíková, E., Melichna, J., \& Nováková, H. (1998). Physiological profiles of male and female taekwon-do (ITF) black belts. Journal of Sports Sciences, 16(3), 243-249. doi: $10.1080 / 026404198366768$

Hopkins, W., Marshall, S., Batterham, A.M. \& Hanin, J. (2009). Progressive statistics for studies in sports medicine and exercise science. Medicine \& Science in Sports \& Exercise, 41(1), 3-12. doi: 10.1249/MSS.0b013e31818cb278

López-Valenciano, A., Ayala F., Vera-Garcia, F.J., De Ste Croix, M., Hernández-Sánchez, S., Ruiz-Pérez, I., Cejudo, A. \& Santonja, F. (en prensa). Comprehensive profile of hip, knee and ankle ranges of motion in professional soccer players. The Journal of Sports Medicine and Physical Fitness (in press).

Markovic, G., Misigoj-Durakovic, M., \& Trninic, S. (2005). Fitness profile of elite Croatian female taekwondo athletes. Collegium antropologicum, 29(1), 93-9.

Pallant, J. (2007). SPSS survival manual: a step by step guide to data analysis using SPSS. 3rd edition. Crows Nest, N.S.W.: Allen \& Unwin.

Pion, J., Fransen, J., Lenoir, M., \& Segers, V. (2014). The value of non-sport-specific characteristics for talent orientation in young male judo, karate and taekwondo athletes. Archives of Budo, 10, 147-150.

Rivera, M., Rivera-Brown, A., \& Frontera, W. (1998). Health related physical fitness characteristics of elite Puerto Rican athletes. Journal of Strength \& Conditioning Research, 12(3), 199-203.

Wasik, J. (2009). Structure of movement of a turning technique used in the event of special techniques in Taekwon-do ITF. Archives of Budo, 5, 111-115.

World Taekwondo Federation. (2017). WTF website. Recuperado de www.worldtaekwondo.org 